\title{
Effect of DHA and CoenzymeQ10 Against $A \beta$ - and Zinc-Induced Mitochondrial Dysfunction in Human Neuronal Cells
}

\author{
Nadia Sadlia Colin J. Barrow ${ }^{\mathrm{b}}$ Sean McGee ${ }^{\mathrm{c}}$ Cenk Suphioglua,b \\ aNeuroAllergy Research Laboratory (NARL), bSchool of Life and Environmental Sciences and \\ 'Metabolic Research Unit (MRU), School of Medicine, Deakin University, Waurn Ponds, Australia
}

\section{Key Words}

Zinc - Docosahexaenoic acid - CoenzymeQ10 - Human neuronal cells • Neuroprotection • Mitochondrial function

\begin{abstract}
Background: Beta-amyloid $(A \beta)$ protein is a key factor in the pathogenesis of Alzheimer's disease (AD) and it has been reported that mitochondria is involved in the biochemical pathway by which $A \beta$ can lead to neuronal dysfunction. Coenzyme Q10 (CoQ10) is an essential cofactor involved in the mitochondrial electron transport chain and has been suggested as a potential therapeutic agent in AD. Zinc toxicity also affects cellular energy production by decreasing oxygen consumption rate (OCR) and ATP turnover in human neuronal cells, which can be restored by the neuroprotective effect of docosahexaenoic acid (DHA). Method: In the present study, using Seahorse XF-24 Metabolic Flux Analysis we investigated the effect of DHA and CoQ10 alone and in combination against $A \beta$ - and zinc-mediated changes in the mitochondrial function of M17 neuroblastoma cell line. Results: Here, we observed that DHA is specifically neuroprotective against zinc-triggered mitochondrial dysfunction, but does not directly affect $A \beta$ neurotoxicity. CoQ10 has shown to be protective against both $A \beta$ - and zincinduced alterations in mitochondrial function. Conclusion: Our results indicate that DHA and CoQ10 may be useful for the prevention, treatment and management of neurodegenerative diseases such as $A D$.
\end{abstract}




\section{Introduction}

The omega-3 fatty acid docosahexaenoic acid (DHA) is the major polyunsaturated fatty acid (PUFA) in the phospholipid fractions of the brain and is required for normal cellular function [1]. Structurally, DHA comprises 22 carbons and six double bonds, making it the most unsaturated fatty acid in cell membranes and important for increasing the fluidity of cell membranes [2]. Increased DHA in cell membranes lowers the susceptibility to lipid peroxidation and oxidative stress in neuronal cells [3]. Therefore, increased intake of DHA is reported to significantly reduce the incidence of neurodegenerative diseases, such as Alzheimer's disease [4, 5]. Previous data has shown that reduced DHA levels in AD brains were associated with an increase in intracellular zinc levels [6], which could suggest a direct interaction between DHA and zinc in the progression of AD. Zinc-mediated brain injury has been implicated as a neurotoxin in models of $\mathrm{AD}$ [7]. However, the mechanism of zinc toxicity is unknown, but evidence suggests that zinc induces cellular apoptosis through inhibition of adenosine triphosphate (ATP) synthesis [8,9], increase in the production of reactive oxygen species (ROS) and eventual loss of mitochondrial membrane potential $\Delta \Psi_{\mathrm{m}}$ [9]. Recently, we reported that DHA protects against zinc-mediated alterations in human neuronal cell bioenergetics and mitochondrial function [10].

$\mathrm{AD}$ is characterized by the accumulation of amyloid-beta $(\mathrm{A} \beta)$-containing plaques, hyperphosphorylated neurofibrillary tangles, neuronal death and synaptic loss $[11,12]$. Impaired mitochondrial function [12] and a decrease in membrane potential $\Delta \Psi_{\mathrm{m}}$ have also been recognized as early events within the $A \beta$ toxicity cascade [13]. Coenzyme Q10 (CoQ10), a component of the mitochondrial electron transport chain, is well characterized as a neuroprotective antioxidant in human neuronal cells [14], as well as in animal models [15] and human trials of AD [16]. CoQ10 has membrane-stabilizing properties and also plays a vital role in ATP production [17]. Like the omega-3 fatty acid DHA, CoQ10 levels are decreased with aging [18], and in a number of neurodegenerative conditions [19].

Both DHA and CoQ10 have demonstrated neuroprotection in human neuronal cells, however it is unclear whether one is better than the other and whether the combined administration of DHA and CoQ10 has additive effects against $\mathrm{A} \beta$ - and zinc-induced mitochondrial dysfunction. In this study, we examined whether combined DHA and CoQ10 could preserve bioenergetics and mitochondrial function better than either DHA or CoQ10 alone, in response to $A \beta$ and zinc toxicity in human neuronal cells.

\section{Materials and Methods}

\section{Cell culture}

Human neuroblastoma M17 cells were cultured in Opti-MEM media (Invitrogen), supplemented with $2.5 \%$ heat inactivated fetal bovine serum (FBS), in a humidified incubator at $37^{\circ} \mathrm{C}$ with $5 \% \mathrm{CO}_{2}$. Trypsin/ EDTA (0.05\%; Gibco) was used for trypsinization.

For treatment analysis, the growth medium was replaced with experimental media (Opti-MEM) treated with and without beta-amyloid $\left(A \beta_{1-42}\right)$ peptide (final concentration $10 \mathrm{nM}$ ), zinc (final concentration $5 \mu \mathrm{M}$ ), Coenzyme Q10 (CoQ10) dissolved in acetone (final concentration $10 \mu \mathrm{M}$ ) and docosahexaenoic acid (DHA) (final concentration $30 \mu \mathrm{M}$; Nu Chek Prep Inc, Elysian, Mn, USA), in the presence of anti-oxidant vitamin E (final concentration $50 \mathrm{nM}$ ).

In order to confirm the extracellular zinc uptake, we have previously tested whether zinc enters M17 human neuroblastoma cells. We observed that zinc does in fact get into cells as shown by our ${ }^{65} \mathrm{Zn}$ studies [20]. The effect of $5 \mu \mathrm{M}$ of zinc concentration on cell viability was also tested to confirm the occurrence of apoptosis. Therefore, $5 \mu \mathrm{M}$ was the concentration chosen for our studies, which is within high physiological levels. The growth media was also tested and found to have no detectable levels of zinc.

Stock solution of DHA in ethanol was stored at $-20^{\circ} \mathrm{C}$ and pre-incubated in complete growth medium at $37^{\circ} \mathrm{C}$ overnight to allow protein conjugation. CoQ10 was a generous gift from Prof. Colin Barrow, School of Life and Environmental Sciences, Deakin University. The synthetic $A \beta_{1-42}$ peptide was purchased from Sigma 
Aldrich (MO, USA) dissolved in dimethyl sulfoxide (DMSO) and stored at $-80^{\circ} \mathrm{C}$ until use. $\mathrm{A} \beta_{1-42}$ peptide was pre-incubated in DMSO at $37^{\circ} \mathrm{C}$ overnight prior to experiments to allow aggregation. Unlike water that would promote a quick $\mathrm{A} \beta$ aggregation, dissolving $\mathrm{A} \beta 1_{-42}$ peptide in DMSO delays the protein aggregation, so pre-incubation prior to the experiment is necessary [21]. We initially tested a range of $A \beta_{1-42}$ peptide concentrations on mitochondrial function and cell viability. $10 \mathrm{nM} \mathrm{A} \beta_{1-42}$ peptide in DMSO was chosen as our final concentration, as it induced mitochondrial dysfunction without reducing cell viability. This concentration has previously been used to perturb mitochondrial function in other neuronal cell lines [22].

\section{Seahorse XF-24 Metabolic Flux Analysis}

M17 neuroblastoma cells were cultured in Seahorse XF-24 (Seahorse Bioscience, Billerica, USA) plates at a density of 25,000 cells per well. Cells were treated with, either the control vehicle, $A \beta$ peptide $(10 \mathrm{nM})$, zinc $(5 \mu \mathrm{M})$, DHA $(30 \mu \mathrm{M})$, or CoQ10 $(10 \mu \mathrm{M})$, alone and in combination for $24 \mathrm{~h}$, prior to analysis. On the day of analysis, cells were changed to unbuffered DMEM (DMEM base medium supplemented with $25 \mathrm{mM}$ glucose, $1 \mathrm{mM}$ sodium pyruvate, $1 \mathrm{mM}$ GlutaMax, $\mathrm{pH} 7.4$ ) and incubated at $37^{\circ} \mathrm{C}$ in a non- $\mathrm{CO}_{2}$ incubator for 1 h. The assay protocol consisted of repeated cycles of $2 \mathrm{~min}$ mix, 2 min wait and 4 min measurement periods, with oxygen consumption rate (OCR) and extracellular acidification rate (ECAR) measured simultaneously through each measurement period by excitation of fluorophores for $\mathrm{O}_{2}$ and $\mathrm{H}^{+}$. This gives measurement of oxidative and non-oxidative metabolism, respectively. Three readings were taken after each addition of mitochondrial inhibitor before injection of the subsequent inhibitors. The mitochondrial inhibitors used were the ATP synthase inhibitor oligomycin (final concentration $1 \mu \mathrm{M}$ ), the proton ionophore carbonylcyanide p-trifluoromethoxyphenylhydrazone (FCCP; $1 \mu \mathrm{M}$ ) and the complex III inhibitor antimycin A $(1 \mu \mathrm{M})$. Mitochondrial function parameters were determined using these mitochondrial inhibitor compounds as modulators to determine a number of bioenergetic and mitochondrial function parameters, including basal respiration, ATP turnover rate, proton leak and maximal and spare respiratory capacity. All treatment conditions were analyzed as ten replicates across 3 plates and data were pooled to give average values for each treatment. After the assays, plates were saved and protein concentrations for each well were measured.

Intracellular reactive oxygen species (ROS) production (Amplex Red assay)

Intracellular $\mathrm{H}_{2} \mathrm{O}_{2}$ production in M17 neuroblastoma cells were determined fluorometrically with the Amplex Red reagent (Molecular Probes, Eugene, OR). Oxidation of Amplex Red coupled by horseradish peroxidase (HRP) causes a reduction of $\mathrm{H}_{2} \mathrm{O}_{2}$ which produces the red fluorescent oxidation product, resorufin [23]. Reaction buffer (1X) is comprised of $0.05 \mathrm{M}$ sodium phosphate (pH 7.4) and was prepared from a $5 \mathrm{X}$ concentrate supplied by the kit. A $10 \mathrm{mM}$ stock solution of Amplex Red was prepared by dissolving Amplex Red reagent in dimethyl sulfoxide (DMSO). Several stock solutions were prepared according to the assay kit instructions. Horseradish Peroxidase (HRP) stock solution $(10 \mathrm{U} / \mathrm{ml})$ was prepared by dissolving HRP powder in $1 \mathrm{X}$ reaction buffer.

M17 neuroblastoma cells were seeded into black well/clear bottom 96-wells plate at 25,000 cells/ well (Corning Costar). Concentrations of each treatment were prepared and $50 \mu \mathrm{l}$ of each treatment was added to the wells. The reaction was initiated by the addition of $50 \mu$ of a working solution that contained $0.1 \mathrm{mM}$ Amplex Red reagent and $0.2 \mathrm{U} / \mathrm{ml} \mathrm{HRP}$ in $1 \mathrm{X}$ reaction buffer. This resulted in a final concentration of $50 \mu \mathrm{M}$ Amplex Red reagent and $0.1 \mathrm{U} / \mathrm{ml}$ of HRP along with the various treatments in the reaction mix with a final reaction volume of $100 \mu \mathrm{l}$ per well. Using a $544 \mathrm{~nm}$ excitation and a $590 \mathrm{~nm}$ emission, fluorescence was measured kinetically every 30 sec for 30 min using a FlexStation II 384 plate reader (Molecular Devices, Sunnyvale, CA). Results were analyzed by the Softmax Pro V5 software.

\section{JC-1 assay (inner mitochondrial membrane potential, $\Delta \Psi_{m}$ )}

Membrane potential across the inner mitochondrial membrane was estimated using the fluorescent indicator dye JC-1 (Invitrogen). M17 neuroblastoma cells were seeded into black well/clear bottom 96-well plate at 25,000 cells/well, followed by incubation of Ab (1 nM), zinc (5 $\mu \mathrm{M})$, DHA $(30 \mu \mathrm{M})$ or CoQ10 $(10 \mu \mathrm{M})$ alone and in combination for $24 \mathrm{~h}$. Stock solution of JC-1 was made up by dissolving JC-1 powder in DMSO at a final concentration of $5 \mathrm{mg} / \mathrm{ml}$ (kept in $-20^{\circ} \mathrm{C}$ ). For a working solution, an aliquot of JC- 1 dye was diluted in 1:25 OptiMem media. $5 \mu \mathrm{l}$ of the working solution was added to each well and incubated in the dark for $10 \mathrm{~min}$ at $37^{\circ} \mathrm{C}$. Cells were washed three times with $1 \mathrm{x}$ phosphate buffered saline (PBS). $100 \mu \mathrm{l}$ of clear 


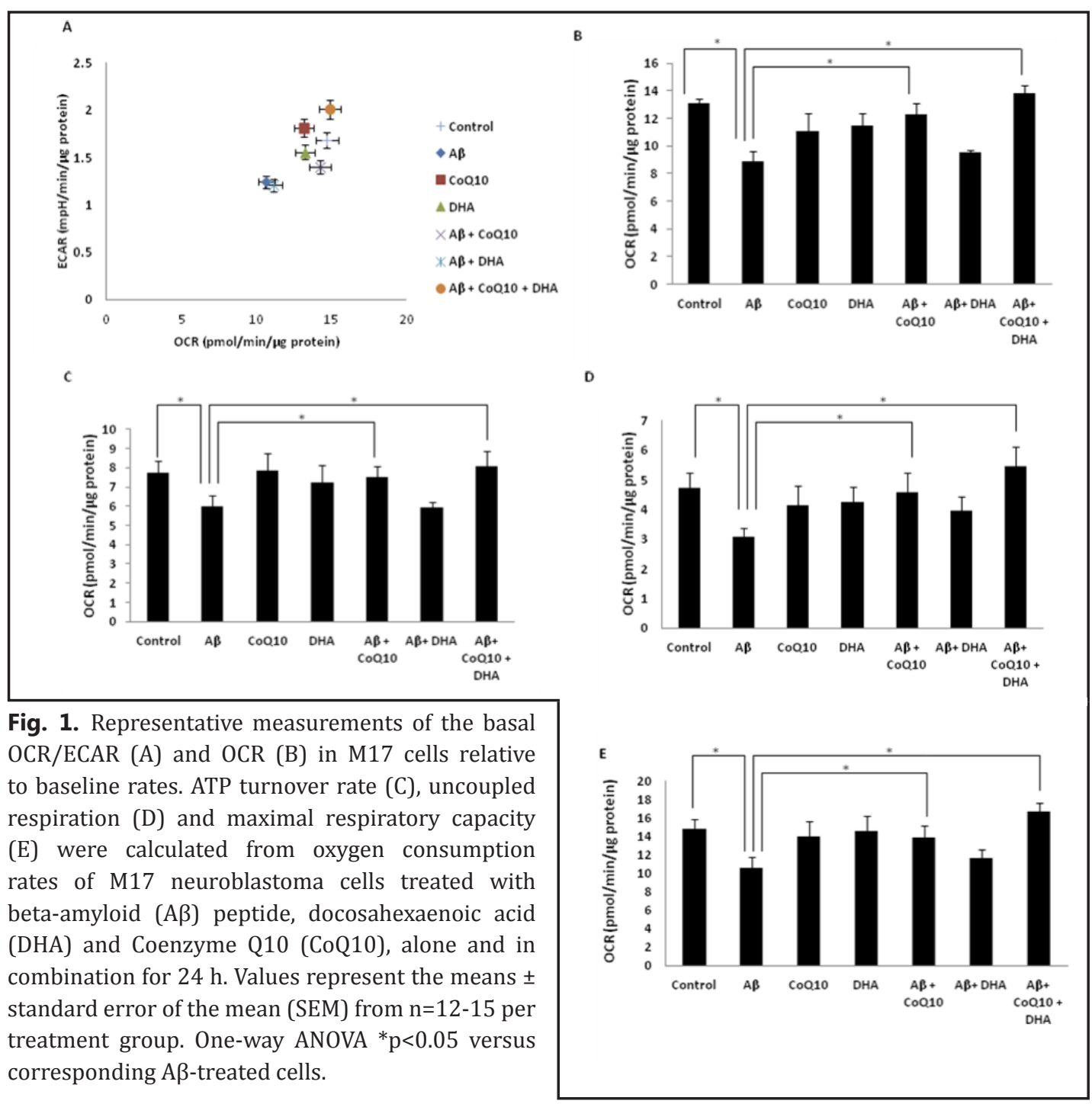

Dulbecco's Modified Eagle Medium (DMEM) without serum was then added in each well and fluorescence was measured using a FlexStation plate reader and analyzed using SoftMax Pro V5 software at $488 \mathrm{~nm}$ excitation and $522 \mathrm{~nm}$ and $605 \mathrm{~nm}$ emissions.

\section{Statistics}

Data were presented as mean and standard error of the mean (SEM). When a one-way ANOVA indicated significant differences among groups, Tukey's post hoc test was used to determine the specific pairs of groups that were statistically different. A $p$ value less than 0.05 was considered statistically significant. Analysis was performed with SPSS 16.0 (SPSS Inc. Chicago, IL, USA).

\section{Results}

A $\beta$ induces mitochondrial dysfunction in M17 neuroblastoma cells, which is restored by CoQ10, but not DHA

To examine whether DHA and CoQ10 could protect against A $\beta$-induced mitochondrial dysfunction in M17 neuroblastoma cells, we measured basal OCR as well as the combined OCR and ECAR response, which produce a bioenergetic function indicative of both mitochondrial respiration and glycolysis, respectively. 


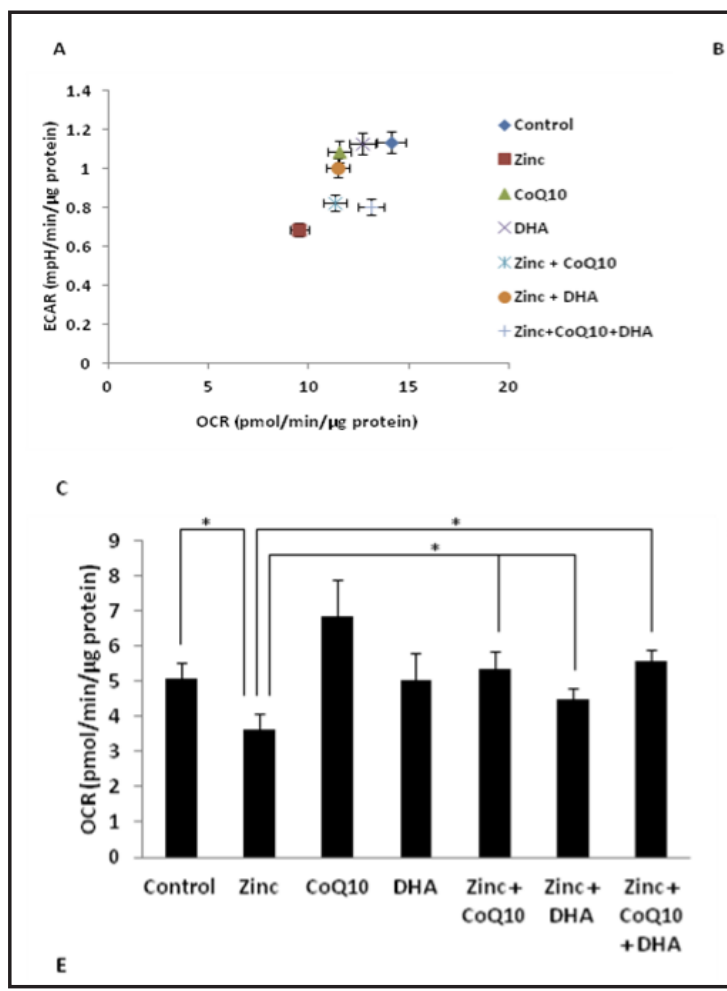

Fig. 2. Zinc-effect on mitochondrial function and basal bioenergetics in M17 cells. Basal OCR/ECAR (A) and OCR (B), as well as ATP turnover rate (C), uncoupled respiration (D) and maximal respiratory capacity (E) were calculated based on oxygen consumption rates of M17 cells exposed to zinc, docosahexaenoic acid (DHA) and Coenzyme Q10 (CoQ10), alone and in combination for $24 \mathrm{~h}$. Values represent the means \pm SEM from $n=12-15$ per treatment group. One-way ANOVA, ${ }^{*} \mathrm{p}<0.05$ versus corresponding zinc-treated cells.
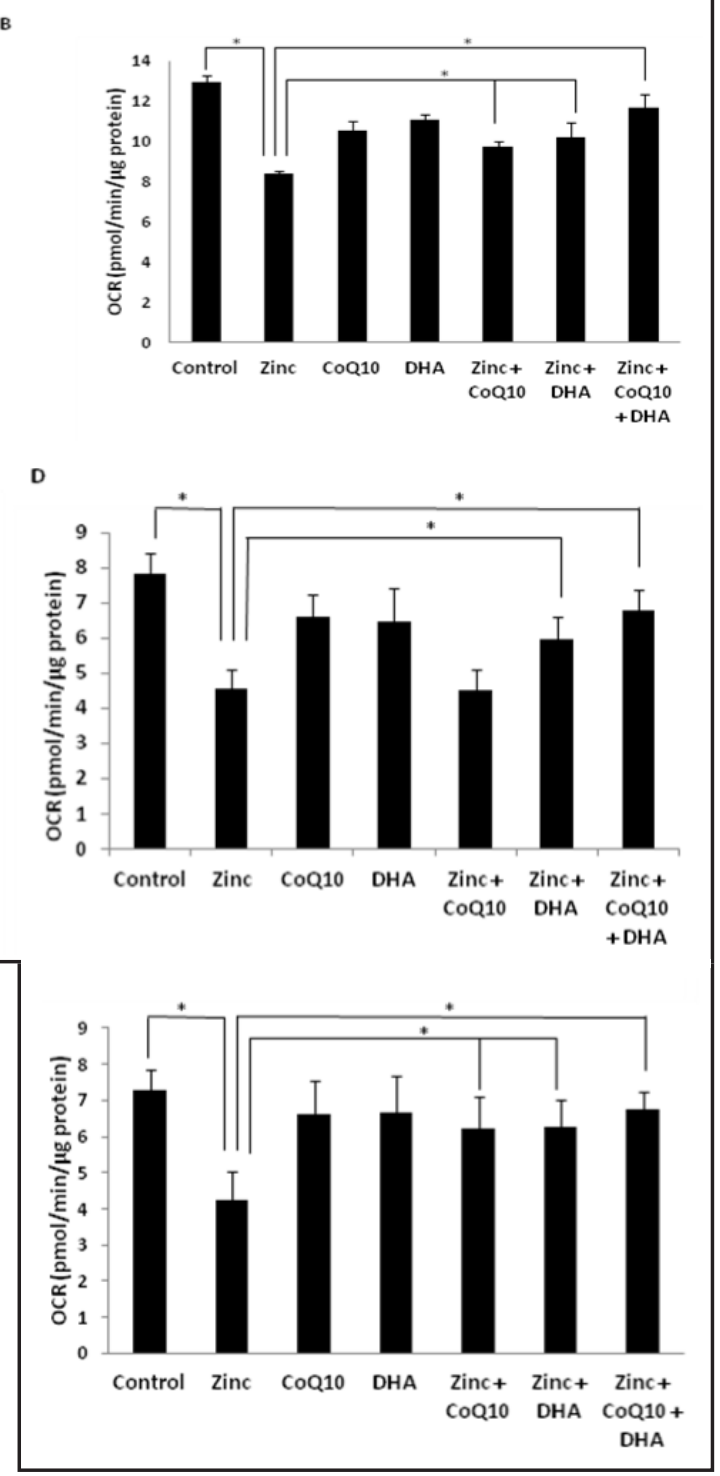

$A \beta$ significantly reduced basal OCR when compared with control cells without altering basal ECAR (Fig. 1A), indicating mitochondrial dysfunction withoutstimulating compensatory glycolysis. A reduction of OCR in response to $A \beta$ treatment was restored by both CoQ10 alone and in combination with DHA (Fig. 1B). However, DHA alone did not directly restore A $\beta$ mediated alteration in basal OCR (Fig. 1B). Similar results were observed for mitochondrial respiration due to ATP turnover (Fig. 1C), uncoupled respiration (proton leak) (Fig. 1D) and maximal respiratory capacity (Fig. 1E).

Zinc impairs mitochondrial bioenergetic function in M17 neuroblastoma cells, which is restored by CoQ10 and DHA, alone and in combination

There was a significant zinc-induced decrease of the basal mitochondrial respiration in M17 cells without alteration in glycolytic rate (Fig. 2A). A significant reduction of OCR in response to zinc treatment was restored by CoQ10 and DHA, alone and in combination (Fig. 2B). The decrease in ATP turnover rate (Fig. 2C), and maximal respiratory capacity (Fig. 2E), were also restored by DHA and CoQ10, either alone or in combination. A decrease in uncoupled respiration was observed in zinc-treated cells, which was restored with either DHA alone and in combination with CoQ10. CoQ10 alone did not significantly affect uncoupled respiration (Fig. 2D). 


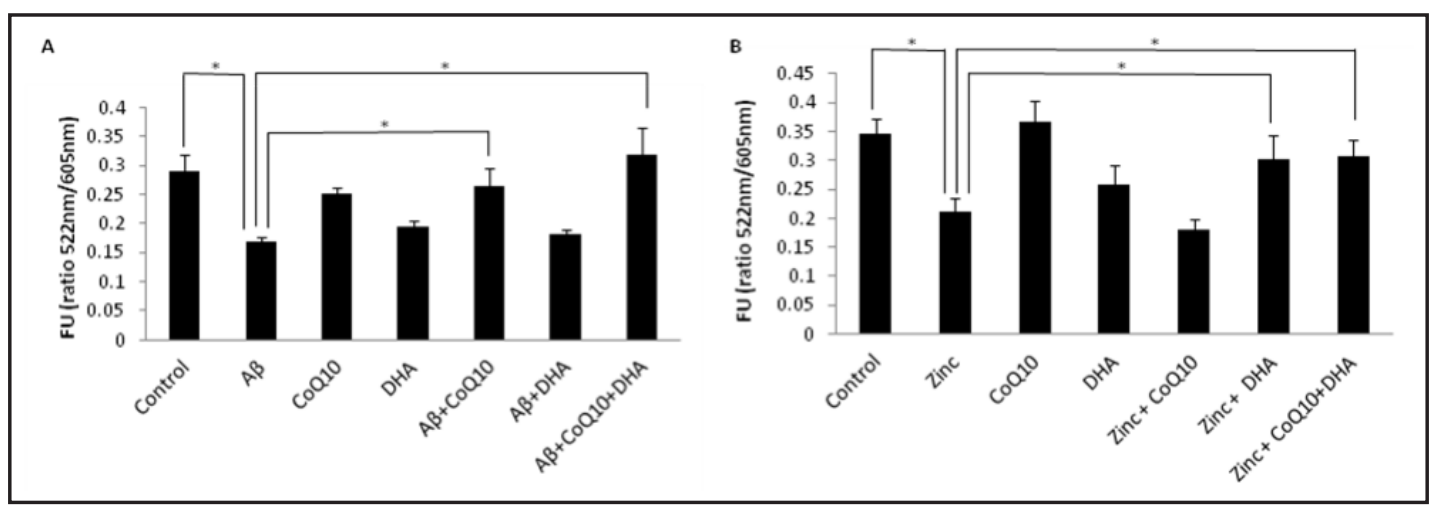

Fig. 3. A $\beta$ - and zinc-effect on the mitochondrial membrane potential $\Delta \Psi_{\mathrm{m}}$ in M17 neuroblastoma cells. Effect of $A \beta$ and zinc on the membrane depolarization of M17 cells' mitochondria were measured by fluorescence plate reader using JC- 1 assay. A $\beta$ - (A) and zinc- (B) treated cells showed a reduction in $\Delta \Psi_{\mathrm{m}}$ compared to the control. The cells were exposed to $A \beta$ and zinc with and without DHA and CoQ10 for $24 \mathrm{~h}$. Data represent mean and SEM of $n=8$ per treatment group with asterisks denoting significant differences between $A \beta$ or zinc alone and co-exposure of DHA/CoQ10-treated cells. One-way ANOVA, ${ }^{*} \mathrm{p}<0.05$ versus corresponding $\mathrm{A} \beta$ or zinc-treated cells.

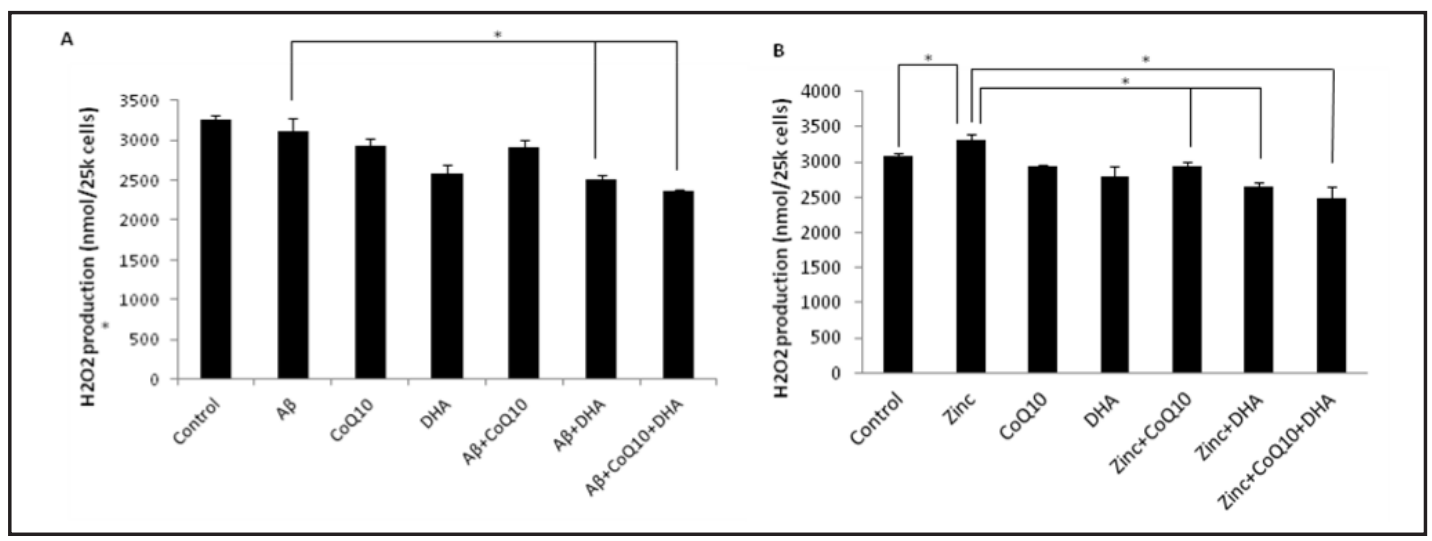

Fig. 4. Neuroprotective effect of DHA and CoQ10 against $A \beta$ - and zinc-induced $\mathrm{H}_{2} \mathrm{O}_{2}$ productions. The Amplex Red assay to quantify ROS productions were performed in the absence/presence of A $\beta$, zinc, DHA and CoQ10, alone and in combination. A $\beta$ (A) did not affect ROS production, whereas zinc (B) significantly increased ROS production in M17 cells. Data are expressed as mean and SEM of $n=8$ per treatment group with asterisks denoting significant differences between $A \beta$ or zinc alone and co-exposure of DHA/CoQ10treated cells.

The neuroprotective effect of DHA and CoQ10 against $A \beta$ - and zinc-induced dissipation of the mitochondrial membrane potential $\Delta \Psi_{m}$ in M17 neuroblastoma cells

We determined the change in $\Delta \Psi_{m}$, alterations in which can contribute to mitochondrial dysfunction [24]. A significant reduction in $\Delta \Psi_{\mathrm{m}}$ following treatment with $A \beta$ was restored by CoQ10, alone and in combination with DHA (Fig. 3A). DHA alone could not restore reduction in $\Delta \Psi_{\mathrm{m}}$ caused by $\mathrm{A} \beta$. Zinc reduced $\Delta \Psi_{\mathrm{m}}$, which was restored by the addition of DHA, but not CoQ10 (Fig. 3B). There were no additive effects of DHA and CoQ10 co-incubation above that with DHA alone, suggesting that CoQ10 was not protective against zinc-mediated alterations in $\Delta \Psi_{\mathrm{m}}$.

Zinc-induced mitochondrial dysfunction is associated with increased production of reactive oxygen species (ROS)

Altered $\Delta \Psi$ m and increase in intracellular levels of $\mathrm{H}_{2} \mathrm{O}_{2}$ in neuronal cells are highly associated with cell apoptosis and the progression of AD. We next evaluated whether DHA 
and CoQ10 could protect against oxidative stress induced by $\mathrm{A} \beta$ and high physiological levels of zinc. We found that $A \beta$ did not affect ROS production when compared to the control cells (Fig. 4A), whereas zinc induced a small but statistically significant increase in $\mathrm{H}_{2} \mathrm{O}_{2}$ levels in M17 neuroblastoma cells (Fig. 4B). However, both DHA and CoQ10, as potent antioxidants, have shown to inhibit $\mathrm{H}_{2} \mathrm{O}_{2}$ production (Figs. $4 \mathrm{~A}$ and $4 \mathrm{~B}$ ).

\section{Discussion}

$A \beta$ and zinc impair basal respiration without alteration in glycolytic rate

To identify the mode of action of both CoQ10 and DHA on A $\beta$ - and zinc-mediated bioenergetic alterations, we measured oxygen consumption rate in M17 cells using the Seahorse extracellular flux analyzer. We observed a significant reduction in basal respiration without alteration in glycolytic rate following exposure to $A \beta$ and zinc.

$A \beta$ and zinc treatments lead to impaired cellular bioenergetics and mitochondrial function Our data show that there are significant reductions in basal respiration, mitochondrial ATP turnover rate, uncoupled respiration and maximal respiratory capacity following A $\beta$ and zinc treatments. This defect of the whole mitochondrial respiratory chain may possibly be due to the accumulated dysfunction of one or several mitochondrial chain complexes as a result of mitochondrial $A \beta$, as well as zinc uptake.

Regardless of the dysfunctional features of end-stage cell death, mitochondrial membrane permeabilization is frequently the decisive event between cell survival and death $[13,25]$. Therefore, to further unravel the effect of $A \beta$ and zinc on mitochondrial respiratory dysfunction, we measured potential proxy of $\Delta \Psi_{\mathrm{m}}$ and ROS production in M17 cells. Our study showed the depletion of $\Delta \Psi_{\mathrm{m}}$ following $A \beta$ and zinc treatment. We suggest that these $A \beta$-induced alterations in mitochondrial function are due to the ability of $A \beta$ to permeabilize cellular membrane and therefore enter the mitochondria. A recent study by Pagani and Eckert (2011) has reported the deposition of $A \beta$ in the outer and inner mitochondrial membrane [26], which may explain the increase in membrane permeability. In the outer membrane, $A \beta$ may be present at the site where it could influence the interaction between mitochondria and anti-apoptotic marker Bcl-2 [26]. However, in the inner membrane, A $\beta$ may interact with the important components of enzymatic activity, metabolic or antioxidant mechanisms and therefore inhibit their actions [26]. These interactions of $A \beta$ would then affect mitochondrial respiration that would potentially impair cellular metabolism. $A \beta$ has been shown to directly inhibit complex IV, but increase in complex III [27], which lead to bioenergetic impairment [13]. Taken together, our results suggest that following a decrease in $\Delta \Psi_{\mathrm{m}}$ and in uncoupled respiration, which results in a futile proton cycle, $A \beta$ exhibit an initial defect in mitochondrial function. This may be caused by inhibition of complex IV activity that is translated into a mitochondrial respiration deficiency with diminished ATP synthesis, which cannot be compensated by an increased activity of complex III.

Apart from decreased ATP turnover rate, maximal respiratory capacity and uncoupled respiratory, high physiological level of zinc has been reported to inhibit electron transfer [28], which could result in dissipation of $\Delta \Psi_{\mathrm{m}}$, as observed in this study. This loss of $\Delta \Psi_{\mathrm{m}}$ contributes to the decrease in $\Delta \Psi_{m}$, which has been reported to be associated with release of cytochrome $c$ and apoptosis-inducing factor (AIF) [9]. A $\beta$ did not have much effect on ROS production, whereas zinc significantly increased ROS production, which indicates that zinc directly caused oxidative stress in neuronal cells leading to mitochondrial dysfunction. Zinc has also been shown to instigate $\mathrm{H}_{2} \mathrm{O}_{2}$ production through tricarboxylic acid cycle (TCA) inhibition [29]. It is clear that zinc-mediated neuronal injury requires the mobilization and redistribution of zinc in the brain. Therefore, the strategies that prevent excessive zinc entry into the cells could ameliorate zinc-mediated cell death [30]. 
DHA may be directly protective against zinc-induced mitochondrial dysfunction, but not towards $A \beta$ toxicity

Recently, we have reported that DHA could protect against zinc-altered mitochondrial dysfunction in M17 cells [10]. Part of this effect could be due to the neuroprotective function of DHA in limiting cellular zinc uptake through decreasing ZnT-3 zinc transporter expression levels $[6,20]$, which in turn inhibits zinc toxicity. Our data show the ability of DHA to restore zinc-induced alteration in $\Delta \Psi_{m}$ and $\mathrm{H}_{2} \mathrm{O}_{2}$ production. We believe that these effects are due to the neuroprotective effect of DHA against zinc-induced ROS production caused by oxidative stress, which therefore indirectly stabilizes $\Delta \Psi_{\text {m }}$.

We observed that DHA alone did not significantly restore $A \beta$-mediated defects in the mitochondrial parameters assessed in this study. However, co-incubations of DHA in the presence of CoQ10 significantly restored $A \beta$-induced alterations to the same extent as CoQ10 treatment alone. From these results, we suggest that DHA alone was not directly protective against $A \beta$-induced mitochondrial dysfunction.

The effect of CoQ10 against $A \beta$ - and zinc-induced alteration in mitochondrial physiology

In this study, we observed that CoQ10 could also interact with zinc and restore zincmediated cellular dysfunction. Our finding is supported by a recent study that a decrease in CoQ10 and increase in zinc levels were observed in chronic obstructive pulmonary disease (COPD) patients [31], which probably result from the defense response of organism against zinc-mediated inflammation. There is no direct evidence in relation to zinc and CoQ10 interactions, but our results suggest that CoQ10 may be neuroprotective against zincinduced mitochondrial dysfunction. Our data also show that CoQ10 restores A $\beta$-induced alteration in membrane potential in neuronal cells, which is possibly caused by the ability of CoQ10 to inhibit the opening of mitochondrial permeability transition pore due to $A \beta$ toxicity and therefore stabilize the membrane [14]. CoQ10 has also been reported to inhibit the aggregation of $A \beta$ and therefore prevent its toxicity [32]. This indicates a direct antiamyloidogenic effect of CoQ10 in neuronal cells.

\section{Conclusion}

This study presents novel findings on the effects of CoQ10 and DHA against A $\beta$ - and zinc-mediated bioenergetic alterations in M17 human neuroblastoma cells. Our results have shown that CoQ10 may have direct effect on $A \beta$-induced alteration in mitochondrial function, while DHA has no significant effect against $A \beta$ toxicity. On the other hand, DHA, due to its direct molecular interaction with zinc $[6,20,33]$, may protect against zincmediated mitochondrial dysfunction. CoQ10, however, has also shown to restore zincinduced alteration in mitochondrial physiology to the same extent as DHA, suggesting the neuroprotective effect of CoQ10 against zinc toxicity.

Taken together, the results of our study indicates the potential of dietary benefits of DHA and CoQ10 for zinc- and A $\beta$-induced neuronal cell apoptosis, respectively, which is characteristic of neurodegenerative diseases such as Alzheimer's disease.

\section{Abbreviations}

A $\beta$ (beta amyloid); ATP (adenosine triphosphate); DHA (Docosahexaenoic acid); DMSO (Dimethyl sulfoxide); ECAR (extracellular acidification rate); HRP (Horseradish Peroxidase); OCR (oxygen consumption rate); ROS (reactive oxygen species); TCA (tricarboxylic acid cycle). 


\section{Conflict of Interests}

The authors declare that they have no competing interests.

\section{Acknowledgments}

This work was supported by School of Life and Environmental Sciences, Centre for Chemistry and Biotechnology (CCB) and Molecular and Medical Research (MMR) Strategic Research Centre (SRC). SLM is supported by a NHMRC Career Development Award.

\section{References}

1 Akbar M, Calderon F, Wen Z, Kim HY: Docosahexaenoic acid: a positive modulator of Akt signaling in neuronal survival. Proc Natl Acad Sci USA 2005;102:10858-10863.

-2 Stillwell W, Wassall SR: Docosahexaenoic acid: membrane properties of a unique fatty acid. Chem Phys Lipids 2003;126:1-27.

-3 Zerouga M, Jenski LJ, Stillwell W: Comparison of phosphatidylcholines containing one or two docosahexaenoic acyl chains on properties of phospholipid monolayers and bilayers. Biochim Biophys Acta 1995;1236:266-272.

-4 Calon F, Lim GP, Yang F, Morihara T, Teter B, Ubeda O, Rostaing P, Triller A, Salem N Jr, Ashe KH, Frautschy SA, Cole GM: Docosahexaenoic acid protects from dendritic pathology in an Alzheimer's disease mouse model. Neuron 2004;43:633-645.

5 Morris MC, Evans DA, Bienias JL, Tangney CC, Bennett DA, Wilson RS, Aggarwal N, Schneider J: Consumption of fish and n-3 fatty acids and risk of incident Alzheimer disease. Arch Neurol 2003;60:940946.

-6 Jayasooriya AP, Ackland ML, Mathai ML, Sinclair AJ, Weisinger HS, Weisinger RS, Halver JE, Kitajka K, Puskas LG: Perinatal omega-3 polyunsaturated fatty acid supply modifies brain zinc homeostasis during adulthood. Proc Natl Acad Sci USA 2005;102:7133-7138.

7 Tougu V, Tiiman A, Palumaa P: Interactions of Zn(II) and Cu(II) ions with Alzheimer's amyloid-beta peptide. Metal ion binding, contribution to fibrillization and toxicity. Metallomics 2011;3:250-261.

8 Weiss JH, Sensi SL, Koh JY: $\mathrm{Zn}^{2+}$ : a novel ionic mediator of neural injury in brain disease. Trends Pharmacol Sci 2000;21:395-401.

-9 Dineley KE, Votyakova TV, Reynolds IJ: Zinc inhibition of cellular energy production: implications for mitochondria and neurodegeneration. J Neurochem 2003;85:563-570.

10 McGee SL, Sadli N, Morrison S, Swinton C, Suphioglu C: DHA Protects Against Zinc Mediated Alterations in Neuronal Cellular Bioenergetics. Cell Physiol Biochem 2011;28:157-162.

-11 Bittner T, Fuhrmann M, Burgold S, Ochs SM, Hoffmann N, Mitteregger G, Kretzschmar H, LaFerla FM, Herms J: Multiple events lead to dendritic spine loss in triple transgenic Alzheimer's disease mice. PLoS One 2010;5:e15477.

-12 Leuner K, Hauptmann S, Abdel-Kader R, Scherping I, Keil U, Strosznajder JB, Eckert A, Muller WE: Mitochondrial dysfunction: the first domino in brain aging and Alzheimer's disease? Antioxid Redox Signal 2007;9:1659-1675.

13 Readnower RD, Sauerbeck AD, Sullivan PG: Mitochondria, Amyloid beta, and Alzheimer's Disease. Int J Alzheimers Dis 2011;2011:104545.

14 Li G, Zou LY, Cao CM, Yang ES: Coenzyme Q10 protects SHSY5Y neuronal cells from beta amyloid toxicity and oxygen-glucose deprivation by inhibiting the opening of the mitochondrial permeability transition pore. Biofactors 2005;25:97-107.

15 Dumont M, Kipiani K, Yu F, Wille E, Katz M, Calingasan NY, Gouras GK, Lin MT, Beal MF: Coenzyme Q10 Decreases Amyloid Pathology and Improves Behavior in a Transgenic Mouse Model of Alzheimer's Disease. J Alzheimers Dis 2011;27:211-213. 


\section{Cellular Physiology Cell Physiol Biochem 2013;32:243-252 and Biochemistry

-16 Ogawa O, Zhu X, Perry G, Smith MA: Mitochondrial abnormalities and oxidative imbalance in neurodegenerative disease. Sci Aging Knowledge Environ 2002;2002:pe16.

17 Beal MF: Therapeutic effects of coenzyme Q10 in neurodegenerative diseases. Methods Enzymol 2004;382:473-487.

18 Kalen A, Appelkvist EL, Dallner G: Age-related changes in the lipid compositions of rat and human tissues. Lipids 1989;24:579-584.

-19 Jimenez-Jimenez FJ, Molina JA, de Bustos F, Garcia-Redondo A, Gomez-Escalonilla C, Martinez-Salio A, Berbel A, Camacho A, Zurdo M, Barcenilla B, Enriquez de Salamanca R, Arenas J: Serum levels of coenzyme Q10 in patients with Parkinson's disease. J Neural Transm 2000;107:177-181.

20 Suphioglu C, De Mel D, Kumar L, Sadli N, Freestone D, Michalczyk A, Sinclair A, Ackland ML: The omega-3 fatty acid, DHA, decreases neuronal cell death in association with altered zinc transport. FEBS Lett 2010;584:612-618.

21 Estus S, Tucker HM, van Rooyen C, Wright S, Brigham EF, Wogulis M, Rydel RE: Aggregated amyloid-beta protein induces cortical neuronal apoptosis and concomitant "apoptotic" pattern of gene induction. J Neurosci 1997;17:7736-7745.

22 Kaneko I, Yamada N, Sakuraba Y, Kamenosono M, Tutumi S: Suppression of mitochondrial succinate dehydrogenase, a primary target of beta-amyloid, and its derivative racemized at Ser residue. J Neurochem 1995;65:2585-2593.

-23 Zhou M, Diwu Z, Panchuk-Voloshina N, Haugland RP: A stable nonfluorescent derivative of resorufin for the fluorometric determination of trace hydrogen peroxide: applications in detecting the activity of phagocyte NADPH oxidase and other oxidases. Anal Biochem 1997;253:162-168.

-24 Sompol P, Ittarat W, Tangpong J, Chen Y, Doubinskaia I, Batinic-Haberle I, Abdul HM, Butterfield DA, St Clair DK: A neuronal model of Alzheimer's disease: an insight into the mechanisms of oxidative stress-mediated mitochondrial injury. Neuroscience 2008;153:120-130. Green DR, Kroemer G: The pathophysiology of mitochondrial cell death. Science 2004;305:626-629. Pagani L, Eckert A: Amyloid-Beta interaction with mitochondria. Int J Alzheimers Dis 2011;2011:925050. Rhein V, Baysang G, Rao S, Meier F, Bonert A, Muller-Spahn F, Eckert A: Amyloid-beta leads to impaired cellular respiration, energy production and mitochondrial electron chain complex activities in human neuroblastoma cells. Cell Mol Neurobiol 2009;29:1063-1071.

28 Nicholls P, Malviya AN: Inhibition of nonphosphorylating electron transfer by zinc. The problem of delineating interaction sites. Biochemistry 1968;7:305-310.

29 Gazaryan IG, Krasnikov BF, Ashby GA, Thorneley RN, Kristal BS, Brown AM: Zinc is a potent inhibitor of thiol oxidoreductase activity and stimulates reactive oxygen species production by lipoamide dehydrogenase. J Biol Chem 2002;277:10064-10072.

-30 Kim AH, Sheline CT, Tian M, Higashi T, McMahon RJ, Cousins RJ, Choi DW: L-type Ca ${ }^{2+}$ channel-mediated $\mathrm{Zn}^{2+}$ toxicity and modulation by ZnT-1 in PC12 cells. Brain Res 2000;886:99-107.

31 Tanrikulu AC, Abakay A, Evliyaoglu O, Palanci Y: Coenzyme Q10, Copper, Zinc, and Lipid Peroxidation Levels in Serum of Patients with Chronic Obstructive Pulmonary Disease. Biol Trace Elem Res 2011;143:659-667.

-32 Ono K, Hasegawa K, Naiki H, Yamada M: Preformed beta-amyloid fibrils are destabilized by coenzyme Q10 in vitro. Biochem Biophys Res Commun 2005;330:111-116.

33 Suphioglu C, Sadli N, Coonan D, Kumar L, De Mel D, Lesheim J, Sinclair AJ, Ackland L: Zinc and DHA have opposing effects on the expression levels of histones H3 and H4 in human neuronal cells. Br J Nutr 2010;103:344-351. 